\title{
Prevalência de desnutrição em crianças com tumores sólidos
}

\author{
Malnutrition prevalence in children \\ with solid tumors
}

Adriana GARÓFOLO ${ }^{1}$

Eliana Monteiro CARAN²

Nasjla Saba SILVA ${ }^{2}$

Fábio Ancona LOPEZ3

\section{R E S U M O}

\section{Objetivo}

Avaliar a prevalência de desnutrição entre crianças com tumores sólidos em tratamento num centro oncológico brasileiro.

\section{Métodos}

Foram avaliados 44 pacientes durante o primeiro mês de tratamento, por meio de medidas antropométricas e, de acordo com recomendações da Organização Mundial de Saúde, seguindo os escores-Z de peso/idade, estatura/idade e peso/estatura para o diagnóstico nutricional.

\section{Resultados}

De acordo com os escores-Z de peso/idade, estatura/idade e peso/estatura, encontramos, respectivamente, $16 \%, 7 \%$ e $16 \%$ de desnutrição entre as 44 crianças. De acordo com os diagnósticos de câncer, $27 \%$ dos portadores de tumores cerebrais, $25 \%$ dos pacientes com neuroblastomas e $11 \%$ com tumores de Wilms, apresentaram desnutrição energético-protéica.

\section{Conclusão}

Há uma elevada prevalência de desnutrição na população estudada, que pode estar relacionada à doença, ao tratamento e aos fatores socioeconômicos, como também à falta de um protocolo nutricional para identificar e tratar precocemente a desnutrição em crianças com tumores sólidos.

Termos de indexação: crianças, estado nutricional, neoplasias, nefroblastoma, transtornos da desnutrição infantil.

\footnotetext{
1 Serviço de Nutrição Clínica, Instituto de Oncologia Pediátrica, Departamento de Pediatria, Universidade Federal de São Paulo/Escola Paulista de Medicina. Rua Botucatu, 743, Vila Clementino, 04023-062, São Paulo, SP, Brasil. Correspondência para/Correspondence to: A. GARÓFOLO. E-mail: <adrigarofolo@hotmail.com>

2 Médica Oncologista Pediátrica, Instituto de Oncologia Pediátrica, Universidade Federal de São Paulo/Escola Paulista de Medicina. São Paulo, SP, Brasil.

${ }^{3}$ Disciplina de Nutrologia e Metabolismo, Departamento de Pediatria, Universidade Federal de São Paulo/Escola Paulista de Medicina. São Paulo, SP, Brasil.
} 


\section{A B S T R A C T}

\section{Objective}

To evaluate the malnutrition prevalence in children and adolescents with solid tumors, who received treatment in a Brazilian oncology center.

\section{Methods}

44 patients were evaluated during the first month of the oncology therapy, using anthropometric measures and, according to the World Health Organization criteria, the Z-scores of weight/age, height/age, and weight/ height.

\section{Results}

The Z-scores of weight/age, height/age, and weight/height showed 16\%, 7\% e 16\% of malnutrition, respectively. Prevalence of malnutrition was observed in $27 \%$ of patients with cerebral tumors, $25 \%$ of those with neuroblastomas, and $11 \%$ of those with Wilms tumor.

\section{Conclusion}

The high prevalence of malnutrition in this population, may be associated with the disease, its treatment, and the social and economic factors. Nonetheless, failure to identify nutritional risk, due to the lack of a nutritional protocol, may be another cause of malnutrition in patients with solid tumors.

Indexing terms: children, nutritional status, neoplasms, nephroblastoma, child nutrition disorders.

\section{N T R O D U Ç Ã O}

A desnutrição energético-protéica (DEP) é observada com freqüência em crianças com câncer de alto grau de malignidade ${ }^{1}$. Nas neoplasias disseminadas, chega a alcançar até $50 \%$ dos casos, estando mais associada a determinadas condições, que dependem do tipo, localização e malignidade do tumor, estágio da doença e tipo de terapêutica².

Atualmente, sabe-se que, à revelia da doença propriamente dita, há fatores relacionados ao tratamento, os quais, durante a terapia, determinam o risco nutricional nas crianças e adolescentes com câncer. Tais fatores têm papel importante na etiologia da desnutrição dos pacientes com câncer, e incluem a redução da ingestão alimentar, assim como as alterações no gasto energético e na absorção e metabolismo de nutrientes, além de outras complicações, como toxicidade oral e gastrintestinal, nefrotoxicidade e infecções ${ }^{3}$.

As modalidades terapêuticas mais agressivas, como grandes procedimentos cirúrgicos, a freqüência dos ciclos de quimioterapia e a radioterapia de grandes áreas, provocam o desenvolvimento da $\mathrm{DEP}^{3}$, pois além de complicações, tais como dor, febre, infecções freqüentes ou graves, causam a redução do apetite e aumentam o requerimento energético, agravando o estado de desnutrição ${ }^{4}$.

A DEP ocorre com maior freqüência entre crianças com tumores sólidos em estágios avançados, quando comparadas às crianças com doença localizada ou com leucemia. Observou-se também que, o maior risco para desnutrição, ao diagnóstico ou durante o tratamento, está associado aos tumores gastrintestinais e à terapia muito agressiva ${ }^{5}$.

Em 1991, Smith et al. observaram, ao diagnósticar crianças com câncer, $5 \%$ de prevalência de desnutrição, de acordo com o escore-Z de peso para estatura (P/E) e estatura para idade (E/I). Porém, no mesmo estudo, mas de acordo com a avaliação da circunferência do braço e da prega cutânea triciptal, respectivamente $20 \%$ e $23 \%$ das crianças apresentaram comprometimento nutricional ${ }^{6}$.

Sanchez et al. (1992) encontraram 14\% de pacientes com DEP, calculada pelo percentual da mediana de peso para estatura, sendo que $47 \%$ desses pacientes apresentaram-se com 
valores de fibronectina e pré-albumina abaixo do normal. A fibronectina é uma glicoproteína, cujo papel parece relacionar-se ao aumento da solidez nas fibras do tecido conjuntivo, estando, freqüentemente, ligada à elastina. O papel da pré-albumina, entretanto, é desconhecido. Estas são proteínas de meia vida curta e, por esse motivo, apresentam-se como bons marcadores de alterações rápidas do estado nutricional. Por isso, alguns autores têm preconizado a utilização da pré-albumina sérica como indicador precoce do estado nutricional em crianças com câncer, principalmente nas leucemias ${ }^{7}$.

Embora a prevalência de desnutrição infantil no Brasil esteja diminuindo (6\% em 1997), esta ainda atinge uma porcentagem superior à de outros países, como os Estados Unidos (3,4\% em 1994), cuja distribuição de renda é mais adequada ${ }^{8,9}$. Esse contingente inclui as crianças com câncer e, portanto, pode estar presente no início do tratamento antineoplásico, desfavorecendo o prognóstico, mesmo com o elevado percentual de cura que muitas neoplasias malignas pediátricas têm alcançado'. Além disso, a DEP intra-hospitalar no Brasil apresenta dados alarmantes. Em adultos, o estudo Ibranutri encontrou uma taxa de $48 \%$ de desnutrição total, sendo que $12,5 \%$ dos casos apresentaram DEP grave $^{10}$.

Poucos estudos oferecem dados sobre a ocorrência de desnutrição em crianças e adolescentes com tumores sólidos não-hematológicos, particularmente no Brasil. Esse fato nos fez crer na aplicabilidade desta pesquisa, objetivando avaliar a prevalência de DEP em crianças com tumores sólidos não-hematológicos, atendidas no Instituto de Oncologia Pediátrica/Universidade Federal de São Paulo, durante a fase inicial do tratamento (terapia de indução da remissão clínica da doença).

\section{M É T O D O S}

Foram avaliados pacientes portadores de neoplasias malignas não-hematológicas, no período compreendido entre março de 1998 a janeiro de 2000. Todos os pacientes estavam realizando a primeira fase do tratamento antineoplásico (indução da remissão clínica da doença), tendo realizado, no máximo, um ciclo de quimioterapia. O estudo foi desenvolvido no Instituto de Oncologia Pediátrica do Departamento de Pediatria da Escola Paulista de Medicina/ Unifesp. O critério de inclusão foi idade inferior a 10 anos, com diagnóstico confirmado de neoplasia maligna não-hematológica.

Foram excluídas crianças em uso de esteróides ou hormonioterapia, pacientes recidivados, bem como crianças com doenças pré-existentes não relacionadas à neoplasia ou ao tratamento antineoplásico, que pudessem repercutir no estado nutricional. Também foi considerado, como critério de exclusão, a avaliação nutricional efetuada após trinta dias da admissão ou já no segundo ciclo de quimioterapia.

O estudo recebeu aprovação da Comissão de Ética Médica da Escola Paulista de Medicina/ Unifesp.

A avaliação foi efetuada por meio da anamnese geral (dados gerais: registro e nome do paciente, data de nascimento, idade, data de admissão, diagnóstico do tumor e outros diagnósticos) e do exame clínico-nutricional, o qual incluiu avaliação antropométrica (peso e estatura), de acordo com a metodologia proposta pela Organização Mundial de Saúde (OMS) ${ }^{11}$. Para a classificação do estado nutricional, foram utilizados os critérios da OMS de 1983 para escore-Z de peso para idade (P/I) $)^{12}$ e OMS (1999) para escores-Z de peso para estatura (P/E) ou estatura para idade $(\mathrm{E} / \mathrm{I})^{11}$

Os dados foram tabulados e analisados por meio de valores percentuais e cálculo de médias e desvios-padrão. Os pacientes foram analisados também separadamente, quanto aos diagnósticos do câncer.

\section{RES U LTA DOS}

Foram avaliadas quarenta e quatro crianças portadoras de tumores sólidos com idades entre 
dois meses e dez anos, sendo vinte e duas (50\%) do sexo masculino. Os diagnósticos dos pacientes e o protocolo de tratamento estão demonstrados na Tabela 1.

De acordo com o escore-Z de P/l, E/I e P/E para o diagnóstico nutricional, observou-se, respectivamente, $16 \%, 7 \%$ e $16 \%$ de prevalência de desnutrição entre as quarenta e quatro crianças. De acordo com os diagnósticos de câncer, 27\% dos portadores de tumores cerebrais, $25 \%$ dos neuroblastomas e $11 \%$ dos tumores de Wilms, apresentaram desnutrição energético-protéica (Tabela 2).
A seguir, os resultados serão apresentados separadamente, segundo o diagnóstico de cada tipo de câncer observado no estudo.

\section{Neuroblastomas}

Doze crianças eram portadoras de neuroblastomas, um no estágio III e onze no estágio IV. A idade variou de 2 a 80 meses e 7 (58\%) eram do sexo masculino.

De acordo com a classificação da OMS, 3 em 12 (25\%) crianças deste estudo apresentaram desnutrição energético-protéica: uma delas

Tabela 1. Diagnósticos e esquema de tratamento de 44 crianças portadoras de tumores sólidos não-hematológicos.

\begin{tabular}{|c|c|c|}
\hline \multirow{2}{*}{ Diagnóstico de câncer } & \multicolumn{2}{|c|}{ Casos } \\
\hline & $n$ & $\%$ \\
\hline Tumores cerebrais $(\mathrm{SNC})^{\text {a }}$ & 15 & 34,0 \\
\hline Neuroblastomas III e IV b & 12 & 27,0 \\
\hline Tumor de Wilms II, III e IV ${ }^{c}$ & 9 & 20,0 \\
\hline Tumores ósseos $^{\mathbf{d}}$ & 3 & 6,8 \\
\hline Sarcomas de partes moles (RMS) ${ }^{\mathbf{d}}$ & 2 & 6,8 \\
\hline Sarcoma hepático embrionário d & 1 & 2,3 \\
\hline Carcinoma renal ${ }^{\mathbf{d}}$ & 1 & 2,3 \\
\hline Tumor neuroectodérmico primitivo ${ }^{d}$ & 1 & 2,3 \\
\hline Total & 44 & 100,0 \\
\hline
\end{tabular}

a Astrocitomas= carboplatina, vincristina e cirurgia; Meduloblastoma= ifosfamida, etoposida, radioterapia e cirurgia. Craniofaringioma:= bleomicina e cirurgia; ${ }^{\text {N} N e u r o b l a s t o m a ~ d e ~ a l t o ~ r i s C O=~ C A P-I C E, ~ c i c l o f o s f a m i d a, ~ d o x o r r u b i c i n a, ~ p l a t i n u m ~ e ~ i f o s f a m i d a, ~ c a r b o p l a t i n a, ~ e t o p o s i d a . ~ C i r u r g i a ; ~}$ 'Actinomicina $D$, vincristina, adriblastina, cirurgia e/ou radioterapia; ${ }^{d}$ Outros= miscelânea.

Tabela 2. Casos de desnutrição entre crianças menores de 10 anos $(n=44)$ diagnosticadas pelos escores- $Z$ de peso para idade $(P / l)$, estatura para idade (E/I) e peso para estatura (P/E) (OMS).

\begin{tabular}{|c|c|c|c|c|}
\hline Pacientes com DEP & Escore-Z P/I & Escore-Z P/E & Escore & re-Z E/l \\
\hline 1 & $-2,84(\mathrm{NBT})^{a}$ & $-2,66(N B T)^{a}$ & & - \\
\hline 2 & $-2,04$ (NBT) & - & & - \\
\hline 3 & - & $-2,22$ (NBT) & & - \\
\hline 4 & $-3,73(\mathrm{SNC})^{\mathbf{b}}$ & $-2,81(S N C) \mathbf{b}$ & $-3,31$ & $(\mathrm{SNC})^{\mathrm{b}}$ \\
\hline 5 & $-2,22(S N C)$ & & & - \\
\hline 6 & $-4,93(\mathrm{SNC})^{\mathrm{c}}$ & $-3,29(\mathrm{SNC})^{\mathrm{c}}$ & $-3,52$ & $(\mathrm{SNC})^{\mathrm{c}}$ \\
\hline 7 & $-5,96(S N C)^{d}$ & $-3,23(S N C)^{d}$ & $-5,11$ & $(S N C)^{d}$ \\
\hline 8 & $-2,34$ (TuWilms) $^{\mathrm{e}}$ & $-2,18$ (TuWilms) & & - \\
\hline 9 & - & $-2,84$ (Osteossarcoma) & & - \\
\hline Total de casos de acordo com cada indicador & $7,00 \quad(15,9 \%)$ & $7,00 \quad(15,9 \%)$ & 3,00 & $(6,8 \%)$ \\
\hline
\end{tabular}

NBT= neuroblastoma; SNC= sistema nervoso central; Tu Wilms= tumor de Wilms; As letras a, b, c, d, e repetidas nas mesmas linhas, significam que aquele paciente foi diagnosticado com desnutrição em mais de um indicador nutricional, simultaneamente. Nove dos $44(20,5 \%)$ pacientes apresentaram uma ou mais de uma das três formas de déficit nutricional (P/l, P/E ou E/I). 
apresentou déficit pelo escore-Z de P/E e P/I, simultaneamente; as outras duas crianças apresentaram desnutrição por um dos indicadores, uma pelo P/l e outra pelo P/E (Tabela 2).

\section{Tumores do sistema nervoso central (SNC)}

Quinze pacientes foram diagnosticados com tumores do SNC, dez (67\%) do sexo feminino e cinco, do masculino. Dentre esses pacientes, o diagnóstico nutricional por meio do escore-Z de $\mathrm{P} / \mathrm{E}$, apresentou uma criança com desnutrição moderada (moderate wasting) e duas, com desnutrição grave (severe wasting), sendo que as três tinham comprometimento estatural grave (severe stunting) de acordo com o escore-Z de E/I (Tabela 2). O escore-Z de P/I revelou quatro crianças com desnutrição, sendo que três delas são as mesmas citadas acima.

\section{Tumor de Wilms}

Nove pacientes com tumor de Wilms foram diagnosticados: três no estágio Il, dois no estágio III e quatro crianças no estágio IV. A faixa etária apresentou variação de 7 a 72 meses, com três crianças abaixo de dois anos. Cinco $(55,6 \%)$ pacientes eram do sexo masculino. Uma (11\%) criança foi diagnosticada com DEP, de acordo com a OMS, pelo escore-Z de P/I, e com DEP moderada (moderate wasting) pelo escore-Z de P/E (Tabela 2).

\section{Tumores menos freqüentes no estudo}

Dos oito pacientes, cinco eram do sexo masculino. Uma criança, diagnosticada com osteossarcoma, apresentou déficit nutricional pelo escore-Z de P/E (moderate wasting) (Tabela 2). As demais, com diagnóstico de osteossarcoma (1), rabdomiossarcoma (RMS) (2), sarcoma de Ewing (1), sarcoma hepático embrionário (1), carcinoma renal (1) e tumor neuroectodérmico primitivo (1), eram eutróficas.

\section{I S C U S S Ã O}

As neoplasias malignas da infância são um grupo distinto de doenças, diferindo na localização, tipo histológico, natureza e comportamento biológico do tumor, repercussões, prognóstico, planejamento terapêutico e faixa etária de incidência. Tais diferenças influenciam as condições nutricionais, de tal modo que, alguns pacientes apresentam desnutrição já ao diagnóstico, enquanto outros têm maior risco de desenvolver desnutrição durante o tratamento ${ }^{13}$.

Sabe-se que os portadores de tumores sólidos apresentam maior prevalência de desnutrição ${ }^{14}$. Porém, os estudos que analisaram a condição nutricional de crianças portadoras dessas neoplasias, agruparam diferentes diagnósticos, o que pode dificultar a interpretação dos resultados, principalmente pelas diferenças no risco nutricional.

Elhasid et al. ${ }^{15}$ não realizaram avaliação antropométrica, mas se preocuparam com os marcadores bioquímicos de desnutrição. Os autores observaram, ao diagnóstico de 50 crianças e adolescentes com tumores sólidos, níveis inferiores de albumina em 2,7\% dos pacientes, enquanto que a pré-albumina encontrava-se abaixo dos níveis normais em $36 \%$ deles.

Alguns tumores sólidos são classicamente conhecidos por promoverem intenso catabolismo no hospedeiro e, portanto, a caquexia é detectada ao diagnóstico da doença. Neste grupo estão, principalmente, os carcinomas, que são menos freqüentes na infância ${ }^{16}$. Porém, a perda de peso e a anorexia também podem estar presentes ao diagnóstico de pacientes com câncer pediátrico, mais freqüentemente entre portadores de tumores ósseos metastáticos e neuroblastomas e de tumores de Wilms de estádio avançado ${ }^{5}$.

Usando o escore-Z de P/I, E/I e P/E para o diagnóstico nutricional, encontramos 16,0\%, 7,0\% e $16,0 \%$ de desnutrição entre as 44 crianças, respectivamente. Estes números são elevados no que diz respeito à DEP aguda, comparados aos resultados da Pesquisa Nacional de Demografia e 
Saúde de 1997 (PNDS). Esta observou DEP em $5,2 \%$ da população no Brasil, de acordo com o escore-Z de P/l; e em 1,4\% e 2,6\% da população, respectivamente em São Paulo e no Brasil, de acordo com o escore-Z de P/E. Entretanto, com relação ao déficit crônico (E/I), que ocorreu em 10,5\% da população brasileira, nossos resultados indicam uma prevalência menor deste tipo de DEP ${ }^{17,18}$.

Estudos com crianças oncológicas em países com melhores condições socioeconômicas, também apresentaram resultados diferentes dos nossos. No estudo de Smith et al. ${ }^{6}$ a incidência de desnutrição avaliada pelo escore-Z de P/E e E/I foi de $2 \%$ e $3 \%$, respectivamente. Schiavetti et al. ${ }^{19}$, estudando 19 crianças italianas portadoras de tumores sólidos, demonstraram que $26 \%$ tinham desnutrição pelo P/E durante o tratamento antineoplásico. Entretanto, o corte utilizado foi $90 \%$ do percentil 50. No nosso estudo, utilizando-se esse ponto de corte ( $<90 \%$ da mediana do P/E), a prevalência total de desnutrição entre as crianças foi de $34 \%$.

Comparando aqueles resultados com os obtidos neste estudo, pode-se supor que, além da doença, a pobreza pode apresentar-se como outro fator de risco nutricional.

O maior percentual de desnutrição ocorreu entre os portadores de tumores do SNC (27\%), seguido dos portadores de neuroblastomas (25\%). Ainda, o grau mais acentuado da desnutrição foi encontrado entre portadores de tumores cerebrais.

No presente estudo, entre as cinco crianças menores de dois anos portadoras de tumores do SNC, a segunda neoplasia maligna mais incidente na infância ${ }^{20}$, quatro apresentaram algum grau de desnutrição, sendo que três delas apresentaram déficits de escore-Z de P/I, E/I e P/E, simultaneamente. Estas três crianças pertencem a um grupo de pacientes que apresentam uma doença rara da infância, denominada síndrome de Russell ${ }^{21}$. Duas delas eram desnutridas graves, pela classificação da $\mathrm{WHO}^{11}$ (Tabela 2). Esta síndrome é provocada por tumores da região hipotalâmica anterior, geralmente gliomas de baixo grau de malignidade. Pela localização, provocam alterações hormonais, hidroeletrolíticas, e de balanço energético, entre outros efeitos, levando a distúrbios nutricionais importantes $22-25$. Neste estudo, isso poderia explicar a prevalência de DEP grave neste grupo de crianças com esse tipo de tumor.

O neuroblastoma, que é uma neoplasia maligna originária de células precursoras do sistema nervoso simpático, ocorre, geralmente, em crianças com idade inferior a quatro $a_{n o s^{26}}$. É classicamente conhecido por produzir catecolaminas, responsáveis por alterações no metabolismo, evidenciadas por taquicardia e sudorese ${ }^{27}$. As metástases do neuroblastoma são precoces e freqüentes, portanto, a perda de peso pode estar presente ao diagnóstico, principalmente pela disseminação do tumor ${ }^{25,26}$.

No estudo, os neuroblastomas também apresentaram alta prevalência de DEP. Todos os casos de neuroblastomas incluídos foram diagnosticados como estágios avançados (III e IV) e, portanto, metastáticos ao diagnóstico.

No estudo de Coates et al. (1981), as crianças com neuroblastoma metastático ao diagnóstico e estado nutricional adequado, apresentaram um período maior em remissão da doença e maior tempo de sobrevida, comparadas às crianças desnutridas. Esses pacientes, principalmente quando de alto risco, recebem terapia antineoplásica agressiva, o que também implica em maior risco nutricional, necessitando, portanto, de suporte nutricional específico ${ }^{28}$.

O tumor de Wilms é a neoplasia renal maligna mais comum da infância ${ }^{29}$. Dos nove casos de tumor de Wilms, três eram lactentes e destes, um era desnutrido pelo escore-Z de P/I e $\mathrm{P} / \mathrm{E}$, simultaneamente. Estudos sobre o estado nutricional destes pacientes são escassos. Entretanto, estágios mais avançados da doença ou histologia desfavorável estão associados com o aumento do risco nutricional ${ }^{5}$.

Assim, entre os grupos estudados, os portadores de tumores cerebrais e neuroblastomas apresentaram as prevalências mais altas de 
desnutrição. Analisando as possíveis causas para o elevado percentual de desnutrição neste grupo de estudo, podemos levantar algumas hipóteses. Além da própria doença, que por si só aumenta o gasto de energia, consome os nutrientes do hospedeiro, aumentando seu catabolismo e prejudicando o metabolismo e aproveitamento normal dos nutrientes, o tratamento tem um impacto extremamente agressivo para o organismo ${ }^{3}$. Estes fatores são inerentes à doença e à terapia antineoplásica e, portanto, relativamente comuns entre os pacientes com câncer. Por outro lado, outros fatores são observados, especialmente nos países em desenvolvimento, como a pobreza e a falta de condições dignas de educação e saúde, as quais podem agravar a situação de risco nutricional ${ }^{6}$.

Os pacientes que integraram este estudo, não foram estudados desde o início dos sintomas até a admissão ao nosso serviço; isto porque, freqüentemente, eles são avaliados por vários profissionais em outros centros de saúde, antes do seu diagnóstico definitivo e da primeira consulta oncológica. Nos casos deste estudo, a avaliação nutricional foi efetuada até um mês após a admissão da criança no nosso serviço; intervalo de tempo que pode ser definitivo para o agravo do estado nutricional. O que nos leva a crer que, a ausência de protocolos de avaliação e terapia nutricional, bem como o limitado envolvimento dos profissionais com a intervenção nutricional precoce, sejam fatores relevantes para explicar a alta taxa de desnutrição encontrada no estudo. Atualmente, os investimentos na área de nutrição em países desenvolvidos, permitem que a intervenção nutricional seja efetuada precocemente e, também por isso, as taxas de desnutrição tendem a ser mais baixas.

Na nossa experiência, a avaliação e terapia nutricional protocoladas, por meio de suplementos orais e sondas, demonstraram recuperar o estado nutricional de crianças e adolescentes com câncer, permitindo o restabelecimento da terapia antineoplásica ${ }^{30,31}$.
O que foi aqui discutido, não deixa dúvidas de que o diagnóstico e a intervenção nutricional precoces devem ser metas prioritárias das equipes que tratam pacientes com câncer, na tentativa de solucionar, pelo menos parte do problema, em curto prazo.

Assim, a implantação de protocolos de terapia nutricional, adaptados à realidade da nossa população, deve ser efetuada para que, no mínimo, esses pacientes tenham a chance de receber o tratamento planejado, evitando-se a redução na dose das drogas, o atraso dos ciclos de quimioterapia ou da cirurgia, e o aumento do risco de toxicidades, infecções e morte.

Os resultados apresentados neste estudo são limitados, considerando o número de pacientes envolvidos. Entretanto, esses dados preliminares podem ser utilizados para estimular e justificar o desenvolvimento de pesquisas multicêntricas, que contribuam para o conhecimento das condições nutricionais de crianças tratadas por câncer em nosso país.

\section{REFERÊ NCIAS}

1. Moreira LAC, Brandão MR, Boente PC. Suporte nutricional em pacientes pediátricos com câncer. Rev Bras Nutr Clin. 1997; 12:55-9.

2. Harrison LE, Brennan MF. The role of total parenteral nutrition in the patient with cancer. Curr Probl Surg. 1995; 32(10):833-924.

3. Mauer AM, Burgess JB, Donaldson SS, Rickard KA, Stallings VA, von Eys J, et al. Special nutrition needs of children with malignancies: a review. JPEN. 1990; 14(3):315-24.

4. Holcomb GW, Ziegler MM. Nutrition and cancer in children. Surg Ann. 1990; 22:129-41.

5. Rickard KA, Grosfeld JL, Coates TD, Weetman R, Baehner RL. Advances in nutrition care of children with neoplastic diseases: a review of treatment, research, and application. Continuing Educ. 1986; 86(12):106-76

6. Smith DE, Stevens MCG, Booth IW. Malnutrition at diagnosis of malignancy in childhood: common but mostly missed. Eur J Ped. 1991; 150(5): 318-22.

7. Sanchez MC, Iraola GA, Gutierrez NA, Altuna MS, Regato JLB. Estudio nutricional en niños oncológicos. An Esp Pediatr. 1992; 36(4):277-80. 
8. Monteiro C, Conde WL, Popkin BM. What has happened in terms of some of the unique elements of shift in diet, activity, obesity, and other measures of morbidity and mortality within different regions of the world? Is obesity replacing or adding to undernutrition? Evidence from different social classes in Brazil. Pub Health Nutr. 2002; 5(1A): 105-12.

9. Wang Y, Monteiro C, Popkin BM. Trends of obesity and underweight in older children and adolescents in the United States, Brazil, China, and Russia. Am J Clin Nutr. 2002; 75(6):971-7.

10. Waistzberg DL, Caiaffa WT, Correia ITD. Hospital malnutrition: the Brazilian national survey (IBRANUTRI): A study of 4000 patients. Nutrition. 2001; 17:573-80.

11. World Health Organization. Management of severe malnutrition: A manual for physicians and other senior health workers. Malnutrition in adolescents and adults. Geneva; 1999.

12. Organización Mundial de la Salud. Medición del cambio del estado nutricional. Ginebra: OMS; 1983.

13. Boente PC, Brandão MR, Aguiar ER, Sampaio C. Terapia nutricional em pacientes oncológicos. Rev Bras Nutr Clin. 1997; 12(2):49-54.

14. Weisdorf-Schindele S, Schwarzenberg SJ. Nutritional support of hematopoietic cell recipients. In: Blume KG, Forman SJ, Appelbaum F. Thoma's hematopoietic cell transplantation. 3th ed. Philadelphia; 2004. p.883-93.

15. Elhasid R, Laor A, Lischinsky S, Postovsky S, Arush MWB. Nutritional status of children with solid tumors. Cancer. 1999; 86(1):119-25.

16. Edan C, Malone M, Pritchard, J. Tumores Raros. In: Dángio GJ, Sinniah D, Meadows A, Evans AE, Pritchard J. Pediatria oncológica prática. Rio de Janeiro: Revinter; 1995. p.449-63.

17. Pesquisa Nacional sobre Demografia e Saúde. Rio de Janeiro: BREMFAM; 1997.

18. Taddei JAAC, Colugnati FAB, Rodrigues EM, Sigulem DM, Lopez FA. Desvios nutricionais em menores de cinco anos. São Paulo: Disciplina de Nutrição e Metabolismo Departamento de Pediatria-UNIFESP/EPM. 2002. p.64.

19. Schiavetti A, Fornari C, Guidi R, et al. Prevalenza delle alterazioni dello stato nutrizionale in un campione di pazienti afferenti a un day hospital oncologico pediatrico. Minerva Pediatr. 2001; 53(3):183-8.
20. Silva NS. Tumores de sistema nervoso central. Ped Moderna. 1999; 35(8):635-9.

21. Gropman AL, Packer RJ, Nicholson HS, Vezima LG, Jakacki R, Geyer R, et al. Treatment of diencephalic syndrome with chemotherapy: Grown, tumor response, and long term control. Cancer. 1998; 83(1):166-72.

22. Waga S, Shimizu T, Sakakura M. Diencephalic syndrome of emaciation (Russell's syndrome). Surg Neurol. 1982; 17(2):141-6.

23. Espiner E, Donaldson I, Chapman B, Bergeron C. Diencephalic idiopatic gliosis: an unusual hypothalamic syndrome of dermophaty, diarrhea and growth arrest. J Endocrinol Invest. 1992; 15(8):609-16.

24. West GA, Berger MS, Geyer JR. Childhood optic pathway tumors associated with ascites following ventriculo-peritoneal shunt placement. Pediatr Neurosurg. 1994; 21(4):254-9.

25. Motil KJ. Sensitive measures of nutritional status in children in hospital and in the field. Int J Cancer. 1998; (11Suppl):S2-9.

26. Caran EM. Neuroblastoma. Ped Moderna. 1999; 35(8):624-7.

27. Sinniah D, Evans AE. Neuroblastoma. In: Dángio GJ, Sinniah D, Meadows A, Evans AE, Pritchard. J Pediatria oncológica prática. Rio de Janeiro: Revinter; 1995. p.377-85.

28. Coates TS, Detamore CM, Rickard KA et al. Relation between nutritional status at diagnosis and outcome in Stage III and IV neuroblastoma. Proc Am Assoc Cancer Res. 1981; 22:402.

29. D’Angio GJ. Tumores de Rim. In: Dángio GJ, Sinniah D, Meadows A, Evans AE, Pritchard J. Pediatria oncológica prática. Rio de Janeiro: Revinter; 1995. p.387-400.

30. Garófolo A, Caran EM, Ancona Lopez F, Petrilli AS. Suporte nutricional na caquexia secundária ao câncer do adolescente: relatado de um caso e revisão da literatura. Rev Paul Pediatr. 2001; 19(2):93-101.

31. Garófolo A, Aragão KSM, Maia PS, Ancona Lopez F, Petrilli AS. Aceitação da suplementação oral e resposta sobre o estado nutricional em crianças e adolescentes desnutridos com câncer. Rev Bras Nutr Clin. 2002; 17(1):1-8.

Recebido para publicação em 29 de agosto de 2003 e aceito em 22 de junho de 2004. 